\title{
High concentrations of atmospheric ammonia induce alterations of gene expression in the breast muscle of broilers (Gallus gallus) based on RNA-Seq
}

\author{
Bao $\mathrm{Yi}^{\dagger}$, Liang Chen ${ }^{\dagger}$, Renna Sa, Ruqing Zhong, Huan Xing and Hongfu Zhang ${ }^{*}$
}

\begin{abstract}
Background: High concentrations of atmospheric ammonia are one of the key environmental stressors affecting broiler production performance, which causes remarkable economic losses as well as potential welfare problems of the broiler industry. Previous reports demonstrated that high levels of ammonia altered body fat distribution and meat quality of broilers. However, the molecular mechanisms and metabolic pathways in breast muscle altered by high concentrations of ambient ammonia exposure on broilers are still unknown.

Results: This study utilized RNA-Seq to compare the transcriptomes of breast muscles to identify differentially enriched genes in broilers exposed to high and low concentrations of atmospheric ammonia. A total of 267 promising candidate genes were identified by differential expression analysis, among which 67 genes were up-regulated and 189 genes were down-regulated. Bioinformatics analysis suggested that the up and down-regulation of these genes were involved in the following two categories of cellular pathways and metabolisms: Steroid biosynthesis (gga00100) and peroxisome proliferator-activated receptor (PPAR) signaling pathway (gga03320), which both participated in the lipid metabolism processes.

Conclusions: This study suggests that longtime exposure to high concentrations of aerial ammonia can change fat content in breast muscle, meat quality and palatability via altering expression level of genes participating in important lipid metabolism pathways. These findings have provided novel insights into our understanding of molecular mechanisms of breast muscles exposed to ammonia in broilers. This study provides new information that could be used for genetic breeding and nutritional intervention in production practice of broilers industry in the future.
\end{abstract}

Keywords: RNA-Seq, Breast muscle, Ammonia, Meat quality, Broiler (Gallus gallus)

\section{Background}

Ammonia $\left(\mathrm{NH}_{3}\right)$ is a colorless, highly irritant, alkaline gas which is produced during the decomposition of organic matter by bacterial deamination or reduction of nitrogenous substances [1, 2]. With the development of intensive large-scale poultry industry, air quality in housing is particularly poor [1,3-5]. Ammonia as a major aerial pollutant of poultry buildings

\footnotetext{
* Correspondence: zhanghongfu@caas.cn

${ }^{\dagger}$ Equal contributors

State Key Laboratory of Animal Nutrition, Institute of Animal Sciences,

Chinese Academy of Agricultural Sciences, Beijing 100193, China
}

is causing increasing problems with broiler health and production.

Based on human and livestock safety, the currently recommended exposure limit for ammonia is set at $25 \mathrm{ppm}[1,6]$. However, the levels of ammonia in broiler houses usually exceed $25 \mathrm{ppm}$, and even go high up to $80 \mathrm{ppm}$, when poultry are confined in buildings provided with artificial heat and ventilation (especially in cold winter of northern China) [3]. High concentrations of gaseous ammonia can have adverse effects on production performance [7]. The greatest problem caused by atmospheric ammonia is reduced growth performance, 
which results in lower body weight gain and higher feed conversion ratios. Ammonia toxicity was reported one century ago by Hahn et al. [8]. To date, most studies of ammonia toxicity have focused on the respiratory tract and nervous system in mammals [9-11]. Few detailed studies have been conducted on the breast muscle tissue response to ammonia exposure in poultry.

Exposure to high levels of ambient ammonia is known to have a detrimental effect on broiler production efficiency and meat yields. It has been shown that high concentrations of ammonia $(70 \mathrm{ppm})$ can reduce growth performance and meat quality of broilers [12, 13]. Previous studies in our laboratory have shown that high concentrations of environmental ammonia can alter body fat distribution and lipid metabolism. Among body fat deposition related tissues of broilers, we focus on breast muscles which are the main source of chicken meat. Chicken meat is one of the most popular food commodities in the world and the second most preferred meat by Chinese consumers $[14,15]$. However, few detailed studies have been conducted on the ammonia exposed breast muscle response in poultry and the molecular mechanisms underlying these effects are yet to be investigated.

Based on previous research, we hypothesized that exposure to high concentrations of atmospheric ammonia can confer negative effects on the muscle tissue of broilers via different mechanisms, which requires further study to elucidate. Previously, broiler chickens with different growth performance or fat content were compared using microarrays [16]. With the rapid development of systems-biology approach, high-throughput transcriptome sequencing can be used to discover genes that are functionally active and participate in specific biological processes [17-20]. In particular, Illumina sequencing technology can be used for gene identification with confirmed reliability [21, 22].

Currently, little is known about the alteration of genes in the breast muscle of poultry that have been exposed to high levels of atmospheric ammonia. Therefore, the objectives of the study reported herein were to utilize a transcriptome sequencing procedure to identify differential expressed genes in breast muscle tissues of broilers exposed to high and low concentrations of atmospheric ammonia. In conclusion, our results provide important information towards understanding the biological basis of variation in ammonia exposed broilers, also provide information towards comprehensively understanding the molecular mechanisms and metabolic pathway alteration of the physiological responses to ammonia which are critical to improve poultry production efficiency and welfare.

\section{Methods}

\section{Ethics statement}

This study was undertaken in strict accordance with the Regulations for the Administration of Affairs Concerning
Experimental Animals of the State Council of the People's Republic of China. The protocol was approved by the Committee on Experimental Animal Management of the Chinese Academy of Agricultural Sciences. Before tissue sampling, birds were humanely sacrificed by cervical dislocation. All efforts were made to minimize distress.

\section{Animals and exposure conditions}

Ninety-six 1-day-old Arbor Acres (AA) male broilers were purchased from a commercial hatchery in Beijing (Beijing Arbor Acers Broiler Co., Beijing, China). All birds were housed in individual wire-bottom cages in an environmentally controlled room under standard brooding practices, and given ad libitum access to water and a maizesoybean basal diet during the first 21 days. Then, broilers were divided into two environmentally controlled exposure chambers. Each exposure chamber was a $4500 \times$ $3000 \times 2500 \mathrm{~mm}$ (length $\times$ width $\times$ height) sealed unit, sectioned for housing 48 birds per chamber. The diet during the experiment was formulated to achieve the National Research Council (NRC, 1994) recommended requirements for all nutrients containing $\mathrm{ME}, 12.77 \mathrm{MJ} \mathrm{kg}^{-1}$, and crude protein $19.93 \%$ (Additional file 1: Table S1). The concentrated ammonia was delivered in a whole body animal exposure chamber from days 22 to 42 , and the chambers were computer programmed to have the ammonia concentration as required. Temperature and airflow were controlled during the exposures to ensure adequate ventilation, minimize buildup of animal-generated contaminants (dander, $\mathrm{CO}_{2}, \mathrm{H}_{2} \mathrm{~S}$ ) and to avoid thermal stress [23, 24]. Broilers in the treatment group were exposed to $75 \pm$ $3 \mathrm{ppm}$ (parts per million) ammonia during the experimental period. Broilers in the control group were raised in a separate chamber with ambient ammonia concentration at $3 \pm 3 \mathrm{ppm}$. The concentration of ammonia in both chambers was monitored with a LumaSense Photoacoustic Field Gas-Monitor INNOVA 1412 (Santa Clara, CA, USA) during the entire experiment. The experiment was carried out at the State Key Laboratory of Animal Nutrition in Beijing, China.

\section{Sample collection}

At day 42, all birds were weighed after a $12 \mathrm{~h}$-fasting (12 h food withdrawal) period. Sixteen birds ( 8 broilers each group) were randomly selected for breast muscle sample collection. The chickens were euthanized by manual cervical dislocation and then exsanguinated for tissue sampling. Samples of breast muscle were collected and prepared for carcass traits measurements. Carcass traits were determined in accordance with the method described by Połtowicz and Doktor [25]. Breast muscle was quickly dissected and weighed, then rapidly frozen in liquid nitrogen, and stored at $-80{ }^{\circ} \mathrm{C}$ for further transcriptome (RNA-Seq) and qRT-PCR analyses. 
Total RNA extraction and CDNA library construction

The breast muscle of the 6 randomly selected broilers (3 birds per group) were separately ground in frozen state in liquid nitrogen. Total RNA of breast muscle was isolated with TRIzol reagent (Invitrogen) according to the manufacturer's instructions. The concentration of RNA samples was measured using the NanoDrop 2000 (Nanodrop Technologies, Wilmington, DE). Agilent 2100 Bioanalyzer (Agilent Technologies, Santa Clara, CA) was utilized to assess the integrity of the total RNA (RIN number $>8.0$ ). The total RNA with lowest quality was not used for further study.

A total amount of $5 \mu \mathrm{g}$ RNA per sample was used as input material for the mRNA preparations. Briefly, mRNA was extracted from total RNA using oligo (dT) magnetic beads (Invitrogen) and sheared into short fragments of about 200 bases. These fragmented mRNAs were then used as templates for cDNA synthesis. The cDNAs were then PCR amplified to complete the library. After PCR enrichment, cDNA quantity and quality were assessed using a NanoDrop 2000 spectrophotometer (Nanodrop Technologies, Wilmington, DE) and Agilent 2100 Bioanalyzer (Agilent Technologies, Santa Clara, CA). After construction, the 6 cDNA libraries were normalized, as suggested by the manufacturer, to $10 \mathrm{nmol} \mu \mathrm{l}^{-1}$ using Tris buffer (10 mmol Tris-HCl, $0.1 \%$ Tween 20 , $\mathrm{pH} 8.5)$. Sequencing libraries were generated using the TruSeq RNA Sample Prep kit v2 (Illumina, San Diego, CA) following manufacturer's instructions. The cDNA library was paired-end sequenced using an Illumina HiSeq 2000 platform. The resultant data was deposited in NCBI's Gene Expression Omnibus (GEO) database (GEO accession: GSE84099).

\section{Quality control and primary analysis of reads}

Before read alignment, the quality of raw sequence reads was checked using the FastQC program (http://www. bioinformatics.babraham.ac.uk/projects/fastqc/), clean reads (valid data) were obtained by removing low quality reads (threshold quality, 20; threshold length, $50 \mathrm{bp}$ ) as well as reads containing adapter sequences, ploy- $\mathrm{N}$ and the sequencing primer from the raw data. At the same time, Q30, GC-content and sequence duplication level of the clean data were calculated. The raw data generated from the Illumina Hiseq platform were filtered using a FastQC quality control analysis. All of the downstream analyses were based on high quality clean reads.

\section{Mapping reads to the chicken reference genome}

Sequencing reads from each sample were mapped to the chicken reference genome [Ensembl Galgal4 (GCA 000002315.2)] using the TopHat program. Parameters of TopHat were set to allow only unique alignment to the reference genome. Reads with more than two mismatches were discarded, and concordant mapping for both reads in a pair was required. To obtain the mapping statistics, the alignment BAM files were further examined using RNA-SeQC. Reads were then annotated to reference genes using Bowtie2/Tophat2 software. The differentially expressed genes (DEGs) between control and treatment groups were identified using DESeq software (http://www-huber.embl.de/users/ anders/DESeq/). Genes with a $P$-value of less than $5 \%$ (i.e., pval $<0.05$ ) and fold-change $\geq 2$ were considered significant.

\section{Differential gene expression and functional analysis}

The DEGs were classified for the categories of molecular functions, cellular component and biological process using gene ontology (GO) annotation. The Kyoto Encyclopedia of Genes and Genomes (KEGG) predicted the metabolic pathways of the DEGs. To identify potential common pathways, DEGs were analyzed for biological process and pathway enrichment using DAVID. Pathway enrichment analysis identifies significantly enriched metabolic pathways or signal transduction pathways using the corrected $P$-value $<0.05$ as a threshold of significance.

\section{Confirmation of differential gene expression levels by quantitative real-time PCR (qRT-PCR)}

To validate the repeatability and reproducibility of gene expression data obtained by RNA-Seq in breast muscle of broilers, we performed qRT-PCR on 15 randomly selected genes. Total RNA was isolated by TRIzol reagent (Invitrogen). The first-strand cDNA was synthesized with M-MLV (Promega). Gene specific primers were designed according to the gene sequence using primer premier 5.0, which were synthesized by

Table 1 Effects of atmospheric ammonia on growth performance and body fat distribution in broiler chickens

\begin{tabular}{lcc}
\hline & \multicolumn{2}{l}{ Groups } \\
\cline { 2 - 3 } & \multicolumn{1}{c}{ Control } & \multicolumn{1}{c}{ Treatment } \\
\hline ADG (g/d) & $99.41 \pm 10.08^{\mathrm{a}}$ & $67.92 \pm 12.68^{\mathrm{b}}$ \\
ADFI (g/d) & $158.42 \pm 5.78^{\mathrm{a}}$ & $125.56 \pm 1.50^{\mathrm{b}}$ \\
FCR (g feed/g weight gain) & $1.64 \pm 0.18^{\mathrm{b}}$ & $1.80 \pm 0.25^{\mathrm{a}}$ \\
Breast muscle (\%) & $23.61 \pm 1.06$ & $22.09 \pm 2.28$ \\
Fat in breast muscle (\%) & $1.14 \pm 0.18^{\mathrm{a}}$ & $0.78 \pm 0.14^{\mathrm{b}}$ \\
Liver (\%) & $19.53 \pm 1.60$ & $19.32 \pm 1.90$ \\
Fat in liver (\%) & $5.41 \pm 1.18^{\mathrm{a}}$ & $3.09 \pm 0.17^{\mathrm{b}}$ \\
Abdominal fat (\%) & $1.62 \pm 0.10^{\mathrm{b}}$ & $2.05 \pm 0.45^{\mathrm{a}}$ \\
\hline
\end{tabular}

Values within a row not sharing a common superscript letter indicate significant difference between groups at $P<0.05$. Numbers are mean \pm S.D $A D G$ average daily gain, $A D F I$ average daily feed intake, $F C R$ feed conversion ratio $\mathrm{a}, \mathrm{b}$ within a row indicate significant difference between groups at $P<0.05$ 
Table 2 Summary statistics for sequence quality and alignment information of six breast muscle samples in two groups

\begin{tabular}{|c|c|c|c|c|c|c|}
\hline Sample & C_1 & C_2 & C_3 & T_1 & T_2 & T_3 \\
\hline Group & Control & Control & Control & Treatment & Treatment & Treatment \\
\hline Raw reads & $73,139,016$ & $73,988,890$ & $70,579,138$ & $74,086,562$ & $82,677,190$ & $100,025,614$ \\
\hline Clean reads & $69,375,390$ & $70,089,536$ & $66,879,214$ & $70,162,270$ & $78,368,448$ & $94,553,606$ \\
\hline Valid ratio (\%) & $94.81 \%$ & $94.68 \%$ & $94.71 \%$ & $94.66 \%$ & $94.75 \%$ & $94.49 \%$ \\
\hline Q30 (\%) & $94.90 \%$ & $94.75 \%$ & $94.79 \%$ & $95.04 \%$ & $95.11 \%$ & $94.92 \%$ \\
\hline GC content (\%) & $51.50 \%$ & $51.50 \%$ & $51.50 \%$ & $51.50 \%$ & $51.50 \%$ & $51.50 \%$ \\
\hline Total mapped reads & $49,236,780$ & $49,313,882$ & $47,427,718$ & $50,794,372$ & $57,048,473$ & $69,576,797$ \\
\hline Uniquely mapped reads & $45,869,880$ & $45,818,693$ & $44,268,019$ & $47,090,588$ & $52,708,435$ & $64,514,418$ \\
\hline Multiple mapped reads & $3,366,900$ & $3,495,189$ & $3,159,699$ & $3,703,784$ & $4,340,038$ & $5,062,379$ \\
\hline Mapping rate (\%) & $70.97 \%$ & $70.36 \%$ & $70.92 \%$ & $72.40 \%$ & $72.80 \%$ & $73.58 \%$ \\
\hline
\end{tabular}

Sangon Biotech (Shanghai, China) (Additional file 2: Table S2). qRT-PCR was performed using a SYBR Fast qPCR Master Mix (Takara). The reaction mixtures were incubated in a 96-well plate at $95{ }^{\circ} \mathrm{C}$ for $20 \mathrm{~s}$, followed by 40 cycles of $95{ }^{\circ} \mathrm{C}$ for $3 \mathrm{~s}$ and $60{ }^{\circ} \mathrm{C}$ for $30 \mathrm{~s}$. All measurements were conducted in triplicates. The chicken glyceraldehyde-3-phosphate dehydrogenase $(G A P D H)$ gene was used as an internal control. The $2^{-\Delta \Delta C t}$ method was used to analyze relative RNA expression.

\section{Statistical analysis}

Gene functions and related gene ontology (GO) terms for relevant gene lists were generated using the DAVID bioinformatics database (https://david.ncifcrf.gov/) [26]. The heat map was generated using $\mathrm{R}$ package "gplots" [27], MA and volcano plots were generated using $\mathrm{R}$ package "ggplot2". Data on gene expressions were analyzed by one-way ANOVA (SAS Version 9.2, SAS institute Inc., Cary, NC) and JMP 10 software (SAS Inst. Inc., Cary, NC). A group difference was assumed to be

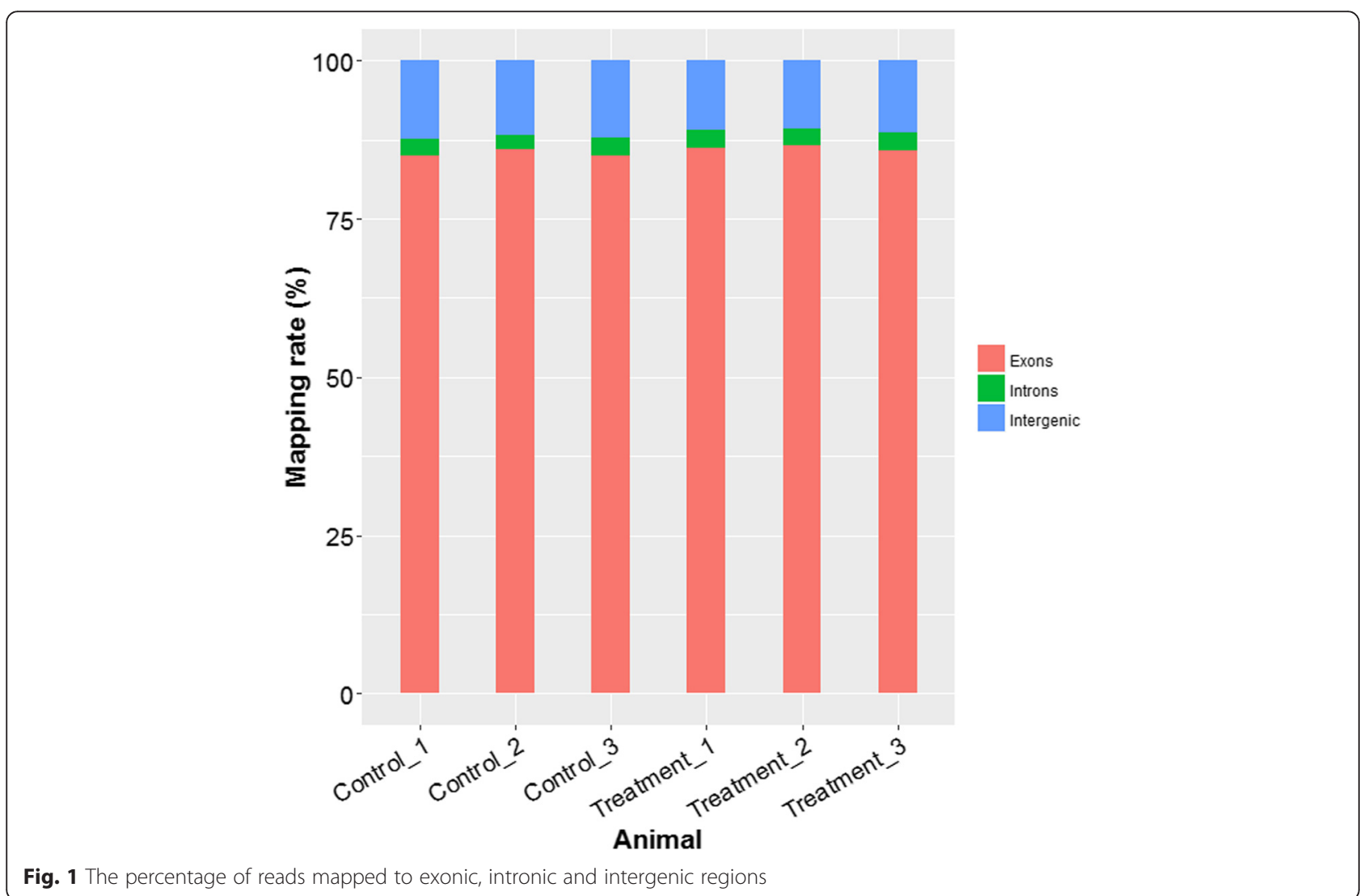


statistically significant when $P<0.05$. All results were expressed as mean \pm S.D.

\section{Results}

Effects of high concentrations of atmospheric ammonia on growth performance and body fat distribution in broilers

In this study, experiments for all birds (control and treatment groups) began on day 22-42. During the entire experimental period (21 days), birds exposed to ammonia had less average daily gain $(\mathrm{ADG})(P<0.05)$ and less average daily feed intake (ADFI) $(P<0.05)$. The feed conversion ratio (FCR) for the treatment group was greatly increased $(P<0.05)$ compared with the control group (Table 1). Ammonia exposure altered body fat distribution of broiler chickens, fat in liver and breast muscle decreased $(P<0.05)$, however, abdominal fat increased significantly $(P<0.05)$ (Table 1$)$.

\section{Overall assessment for mapping statistics}

The RNA-Seq of six breast muscle samples yielded around 474.5 million of raw paired-end reads. After quality filtering, each sample remained approximately 7.5 gigabases $(\mathrm{Gb})$ high-quality sequence data, ranging from 6.7 to $9.5 \mathrm{~Gb}$. Using TopHat2 software, more than $70.36 \%$ of clean reads per sample were mapped back to the reference genome after alignment. Almost 65.37$68.23 \%$ reads were aligned in a unique manner, while $4.72-5.54 \%$ as multiple-mapped reads. The summary of alignment for all samples is shown in Table 2. Among total mapped reads, the vast majority of which (85.01$86.66 \%)$ fell into annotated exons, $10.64-12.31 \%$ was within the large intergenic territory, and only 2.33$2.90 \%$ was located in introns (Fig. 1).

The relative expression of a gene is normalized as fragments per kilobase of transcript per million mapped fragments (FPKM), which is proportional to the number

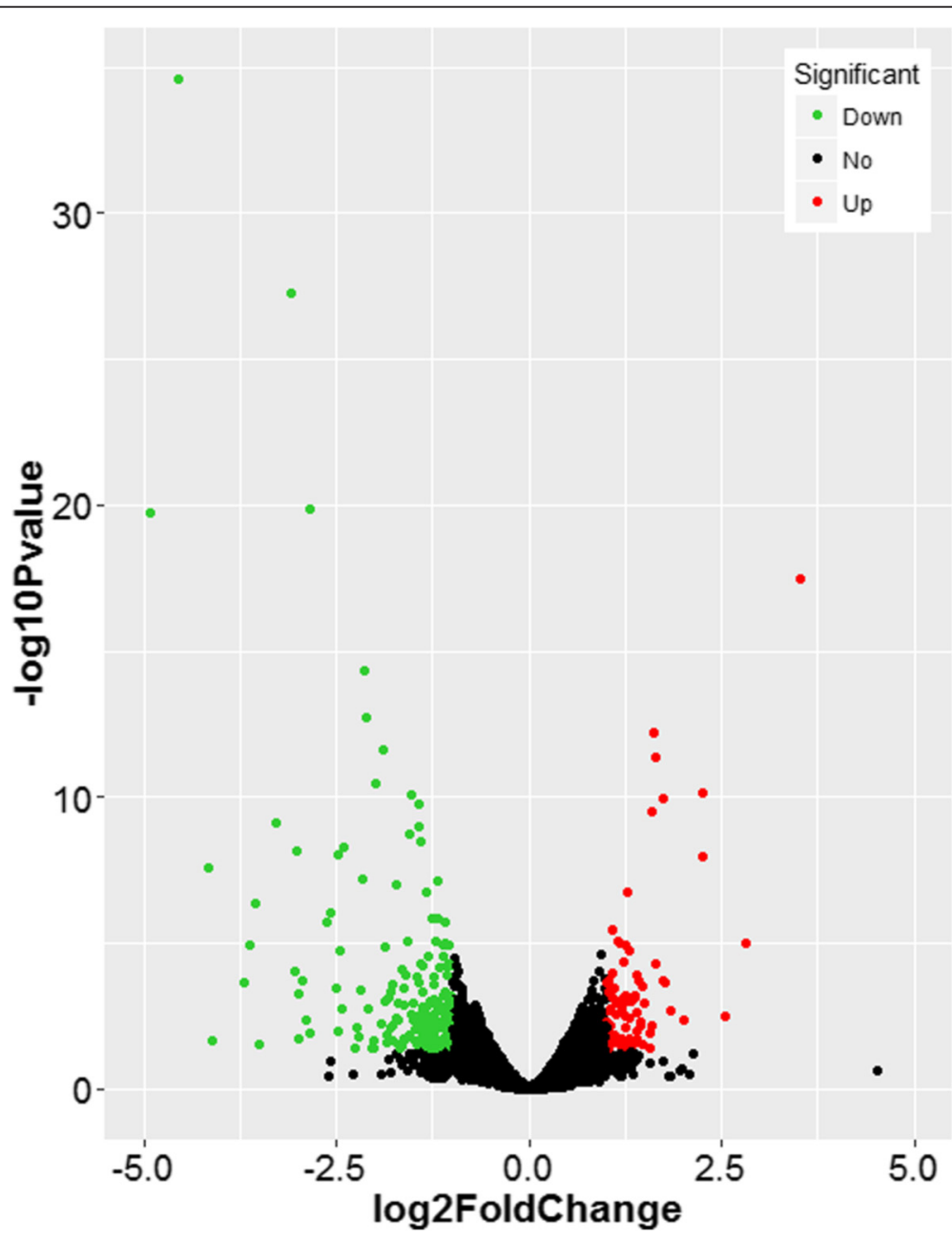

Fig. 2 Volcano plot. Red dots (Up) represent significantly up-regulated genes $(P<0.05$, fold change $\geq 2)$; green dots (Down) represent significantly down-regulated genes $(P<0.05$, fold change $\leq 0.5)$; black dots $(\mathrm{No})$ represent insignificantly differential expressed genes 
of cDNA fragments originated from the gene transcript. The lowest limit of gene expression value is set to be 0.5 FPKM in at least one of the 6 samples. According to this limit, 11,340 genes are identified as being expressed in the breast muscle tissues (Additional file 3: Table S3). The correlation analysis based on the gene expression profiles found that the correlations among 3 samples were greater than 0.92 (Additional file 4: Figure S1).

\section{Gene differential expression analysis}

DESeq software was used for subsequent differential analysis. In total, 267 genes were differentially expressed at a fold-change $\geq$ a space 2 or $\leq 0.5$ at $P<0.05$ in response to high level ammonia exposure (Additional file 5: Table S4 and Fig. 2). Of these genes, 189 genes were down-regulated and 78 were up-regulated (Fig. 2 and Additional file 6: Figure S2). The top ten up and downregulated genes are listed in Table 3 . The fold changes induced by high ammonia ranged from -30.4 to 11.4 (Fig. 3 and Table 3).

\section{Confirmation of RNA-Seq experiment}

To evaluate the reliability of results from RNA-Seq, qPCR was performed using aliquots of non-pooled RNA samples. Fifteen protein-coding genes (FBXO32, TPM2, ASB2, GLUL, CTSL2, ACSL1, CD36, CREB5, PTER, AMPD1, ASB12, KSR1, ACTC1, LIMS1, SNCG) were randomly selected. The results showed a high consistency between RNA sequencing and the qPCR methods (Fig. 4), and confirmed that the relative gene expression of RNA-Seq was reliable.

\section{GO annotations and pathway analysis of differential expressed genes}

Gene ontology (GO) was used to evaluate the function of differentially expressed genes in two groups. All the differentially expressed genes were performed by gene ontology terms through the DAVID platform (Fig. 5). In the biological process category, the genes that participate in cellular development, response to stimulus (carbohydrate and monosaccharide) were at the top ratio in the differentially expressed genes, in the cellular component group, the differentially expressed genes are concentrated in "stress fiber" and "actomyosin" (Fig. 5). The main molecular functional group of differentially expressed genes are related to binding (heparin, low-density lipoprotein and lipoprotein binding) and transcription regulator activity. The KEGG pathway analysis revealed two overrepresented pathways, including steroid biosynthesis and peroxisome proliferator-activated receptor (PPAR) signaling pathway (Fig. 6 and Table 4).
Table 3 Top 10 down- and up-regulated genes in treatment group compared to control group

\begin{tabular}{|c|c|c|}
\hline Gene ID & Gene symbol & Fold change \\
\hline Down-regulated genes & & Down \\
\hline NM_001199909.1 & PDK4 & 30.4 \\
\hline XM_004940680.1 & FHL1 & 23.5 \\
\hline NM_001277411.1 & CA3 & 18.1 \\
\hline NM_001167752.2 & $M B$ & 17.3 \\
\hline XM_004937471.1 & PFKFB3 & 13.0 \\
\hline XM_004937841.1 & $M Y B P C 1^{a}$ & 12.4 \\
\hline XM_416332.4 & $M Y B P C 1^{b}$ & 11.8 \\
\hline NM_001005431.1 & FKBP5 & 11.4 \\
\hline NM_001030956.1 & FBXO32 & 9.8 \\
\hline XM_416965.4 & METTL21C & 8.5 \\
\hline Up-regulated genes & & Up \\
\hline XM_004950318.1 & LOC101748351 & 11.4 \\
\hline XM_419014.3 & SBK2 & 7.0 \\
\hline XM_004938015.1 & CYB5R3 & 5.8 \\
\hline XM_417174.4 & DCUN1D5 & 4.8 \\
\hline NM_204672.1 & SNCG & 4.8 \\
\hline NM_001007477.3 & LOC396531 & 4.0 \\
\hline XM_004944616.1 & LOC770936 & 3.6 \\
\hline XM_419574.4 & C1ORF96 & 3.4 \\
\hline NM_001001766.1 & LIMS1 & 3.4 \\
\hline XM_004946388.1 & DNAJC3O & 3.4 \\
\hline
\end{tabular}

PDK4, pyruvate dehydrogenase kinase, isozyme 4, FHL1 four and a half LIM domains 1 CA3, carbonic anhydrase III, muscle specific, $M B$ myoglobin, PFKFB3 6-phosphofructo-2-kinase/fructose-2,6-biphosphatase 3,MYBPC ${ }^{\text {a }}$ myosin binding protein $\mathrm{C}$, slow type, transcript variant $\mathrm{X} 1, M Y B P C 7^{\mathrm{b}}$ myosin binding protein C, slow type, transcript variant $X 2$, FKBP5 FK506 binding protein 5 , FBXO32 F-box protein 32, METTL21C methyltransferase like 21C, LOC101748351 spindle and kinetochore-associated protein 1-like, SBK2 SH3-binding domain kinase family, member 2, CYB5R3 cytochrome b5 reductase 3, DCUN1D5 DCN1, defective in cullin neddylation 1, domain containing 5, SNCG synuclein, gamma (breast cancer-specific protein 1), LOC396531 parvalbumin, LOC770936 serine/threonine-protein kinase SRPK3-like, C1ORF96 chromosome 3 open reading frame, human C1orf96, LIMS1 LIM and senescent cell antigen-like domains 1, DNAJC30 DnaJ (Hsp40) homolog, subfamily C, member 30

\section{Discussion}

Breast muscle tissue is important in broilers, which is the main source of chicken meat $[28,29]$. Alterations in muscles metabolism pathways induced by longtime ammonia exposure may lead to the decline of meat yields and eating quality $[12,23,30,31]$. In the present study, high-throughput transcriptomic analysis was performed to elucidate the molecular mechanisms in broiler muscles exposed to high and low concentrations of aerial ammonia. Consistent with previous observations, our data showed a negative correlation between ammonia exposure and growth performance in birds $[1,3,7,24]$.

The results obtained in our study indicated that analyzed control and treatment groups differed in the 

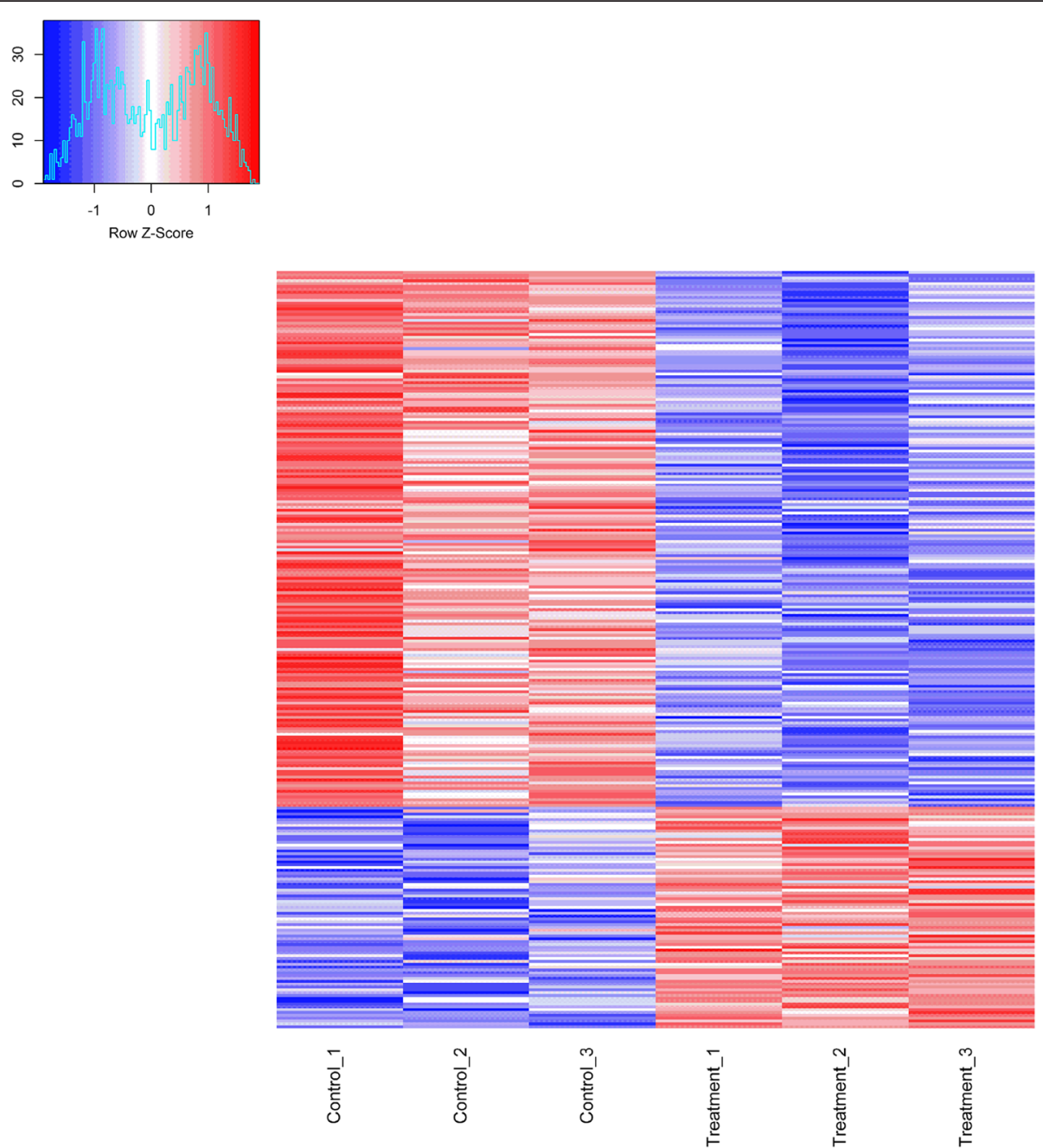

Fig. 3 The relative expression level (in FPKM) of differentially expressed genes between control and treatment groups. The X-axis is sample expression pattern in different concentrations of ammonia exposed groups. The first three samples are in control group; the latter three samples are in treatment group. The $\mathrm{Y}$-axis is the gene clusters across groups

expression of 267 transcripts $(P<0.05)$. As fat content in breast muscles decreased, consistent with our expectations, these differential expressed genes major participated in two lipid metabolism-related pathways (gga00100 and gga03320). Differential expressed genes involved in PPAR signaling pathways here have been proven to be functional in lipid metabolism and fat deposition including CD36 (CD36 molecule), SLC27A1 (solute carrier family 27, member 1), and ACSL1 (acylCoA synthetase long-chain family member 1 ). The above mentioned differential expressed genes in lipid metabolism may contribute to the lower fat deposition in muscles.

It has been reported that both the FAT/CD36 and ACSL facilitate the transport of fatty acids released by lipoprotein lipase [32]. CD36, localizing to the plasma membrane and mitochondria as a fatty acids receptor, functions in fatty acids uptake, transport and oxidation $[33,34]$, and is shown to be highly expressed in skeletal muscle tissues that require energy of fatty acids oxidation [35]. McFarlan et al. found that CD36 knockout mice hindered fatty acids transport, oxidation, and decreased lipid content in muscles [36]. Consistent with that, in the current study, CD36 was 1.5 times lower in high level ammonia exposed group than control group, resulting in lower fat deposition in muscles. Joseph et al. have shown that ACSL1 participates in the biosynthesis of unsaturated fatty acids and fatty acid metabolism, playing a key role in lipid metabolism [37]. In pig breeds, ACSL1 gene might be related to the capacity of fat deposition and meat quality [38]. Therefore, ACSL1 may function an analogous role in broilers in our ammonia 


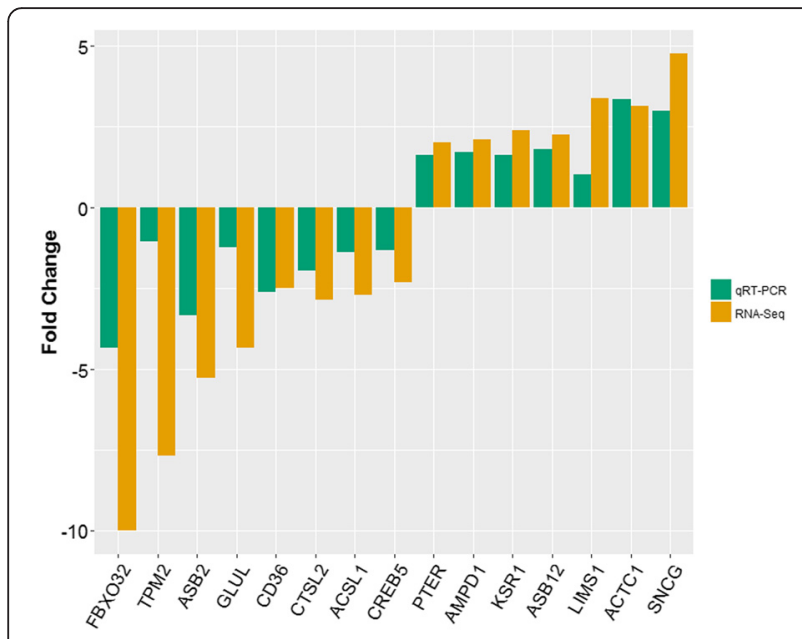

Fig. 4 qRT-PCR validation of differential expressed genes from breast muscle of 42-day-old AA broilers. GAPDH was used as an internal control, and data are presented as fold change ( $n=3$ per group)

exposure experiment. SLC27A1 is another key gene involved in fatty acids transport and lipid metabolism. It is mostly expressed at muscle and adipose tissues, Wu et al. have found that inactivation of SLC27A1 can protect mouse from diet-induced obesity [39, 40]. High level ammonia exposure decreased the expression of SLC27A1 leading to negative effects to adipogenesis in breast muscles may through similar mechanisms provided by $\mathrm{Wu}$ et al. In this study, a high level of ammonia exposure decreased the expression of CD36, ACSL1 and SLC27A1 blocking fat deposition, which is in agreement with the results of above mentioned research.

Lipid metabolism is important in muscle tissue of broilers, which is correlated with meat quality parameters and palatability of consumers [41, 42]. Moreover, high concentrations of aerial ammonia had a detrimental effect on meat tenderness and eating quality [12, 23]. Tenderness is a determinant parameter of meat quality labeled by shear force, Sackett et al. have showed that broilers exposed to $75 \mathrm{ppm}$ ammonia significantly increased shear values in breast muscle, decreased meat tenderness and quality [43]. Taken together, high concentrations of ambient ammonia exposure can cause the decline of tenderness in breast muscle, and lead to decrease of chicken meat quality [12, 44-46]. Previous reports have shown that the gene ASB2 (Ankyrin repeat and SOCS Box containing 2) possess higher expression levels in a high tenderness broiler group than in a low tenderness of chicken group in breast muscles [13, 47], suggesting that it may participate in the tenderization process in broiler chickens. ASB2 was regarded as a participant in the tenderization process in broiler chickens as its product results in the degradation of filamins, proteins which form muscle fibres [12, 48, 49]. In our study, a high level of ammonia exposure decreased the expression of $A S B 2$ which was also confirmed by qRT-PCR.

Besides, in the current research, another differential expressed gene characterized by negative effects on breast meat quality in broilers was also observed. The PLIN2 (perilipin 2) gene has been reported to be involved in the regulation of intracellular lipid storage and fatty mobilization [50]. Conte et al. showed that the high expression of PLIN2 is associated with decreased muscle strength [51]. In pigs, increased PLIN2 mRNA expression was detected in the skeletal muscle of pigs with higher intramuscular fat content [52-54]. As we all know, tenderness and intramuscular fat content both are important parameters of meat eating quality [55-58]. Taken together, we proposed that, in this study, PLIN2

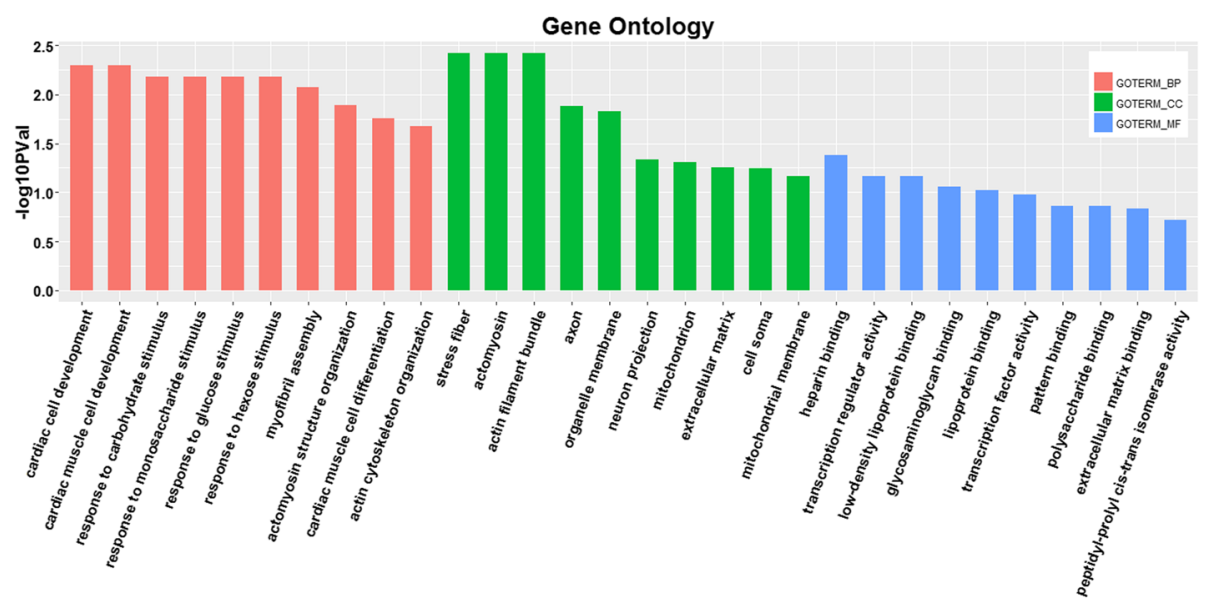

Fig. 5 Gene ontology (GO) annotation of differentially expressed genes. GOTERM_BP, biological process; GOTERM_CC, cellular component; GOTERM_MF, molecular function 


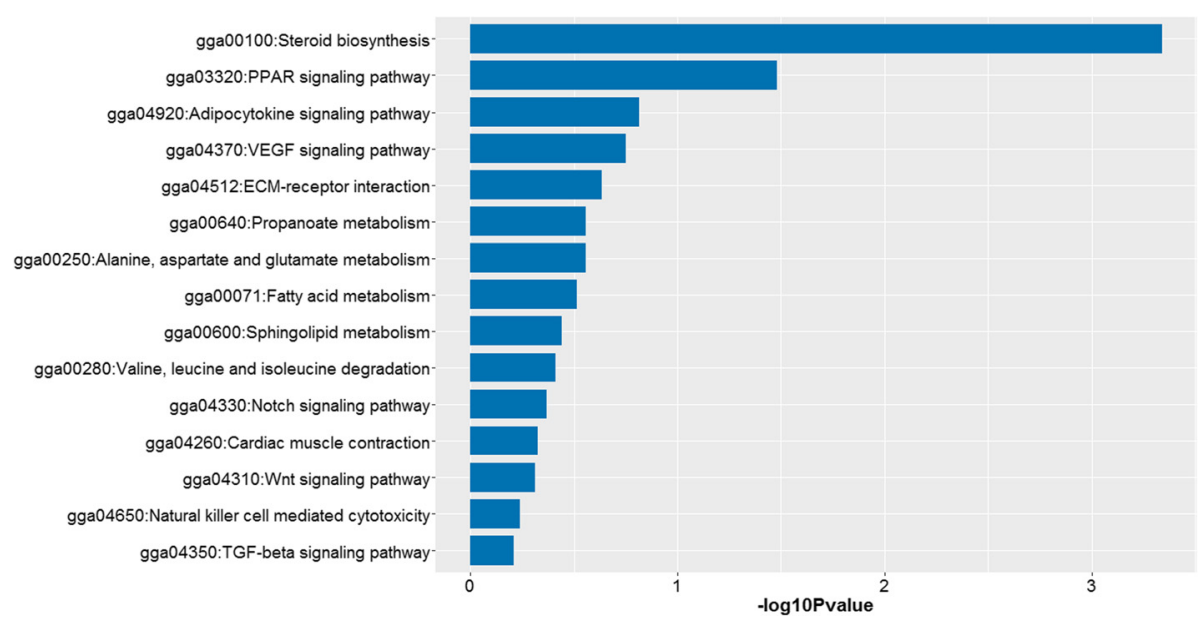

Fig. 6 KEGG Pathway analysis of differentially expressed genes

gene expression abundance altered by ammonia may impair meat quality by increasing shear force meanwhile decreasing fat content in muscles.

In summary, the breast muscle transcriptome analysis demonstrated that broilers exposed to high concentrations of atmospheric ammonia altered body fat content and meat quality through changing the expression abundance of key lipid metabolism genes (such as CD36, ACSL1, SLC27A1, ASB2 and PLIN2) and pathways (gga00100 and gga03320).

\section{Conclusions}

In conclusion, high levels of ammonia resulting in fat content changes in breast muscle tissues can be characterized through high-throughput RNA sequencing analysis. The present transcriptome analysis has demonstrated that exposure to high concentrations of aerial ammonia leads to differential abundances of a number of genes (CD36, ASB2, ACSL1, PLIN2 and etc.) in breast muscles of broilers. To our knowledge, this is the first study of the relationships between gene expression in breast muscle tissues and aerial ammonia exposure in broiler chickens. The ammonia may play a negative role in

Table 4 Significantly enriched pathways for differential expressed genes

\begin{tabular}{llc}
\hline Pathway name & Enriched genes & P-value \\
\hline Steroid biosynthesis & DHCR24, DHCR7, HSD17B7, LSS & 0.00 \\
PPAR signaling pathway & ACSL1, CPT-1A, CD36, SLC27A1 & 0.03
\end{tabular}

DHCR24 24-dehydrocholesterol reductase, $D H C R 7$ 7-dehydrocholesterol reductase, HSD17B7 hydroxysteroid (17-beta) dehydrogenase 7, LSS lanosterol synthase (2,3-oxidosqualene-lanosterol cyclase), ACSL1 acyl-CoA synthetase long-chain family member, CPT-1A carnitine palmitoyltransferase $1 \mathrm{~A}$ (liver), CD36 CD36 molecule (thrombospondin receptor), SLC27A1 solute carrier family 27 (fatty acid transporter), member 1 meat quality by decreasing the lipid content, but the detailed molecular mechanisms of lipid deposition altered by ammonia still need to be further elucidated (perhaps varying lengths of ammonia exposure). The results of our study provide a foundation for future investigations into the gene interactive networks involved in the response to ammonia, and for the development of strategies to avoid the negative impact of ammonia on animal productivity in broiler industry. This study provides theory basis for environmental control (ammonia) and animal welfare in poultry industry.

\section{Additional files}

Additional file 1: Table S1. Composition of the experimental diet and calculated proximate composition of the diet. (DOCX 14 kb)

Additional file 2: Table S2. The GPCR primers used for verification of the differentially expressed genes of the AA broiler breast muscle tissues. (DOCX $17 \mathrm{~kb}$ )

Additional file 3: Table S3. Genes expressed in the breast muscle tissues of broilers are identified using sequencing. (XLSX $2831 \mathrm{~kb}$ )

Additional file 4: Figure S1. The correlation analysis. (TIF $177 \mathrm{~kb}$ )

Additional file 5: Table S4. Differentially expressed genes in chickens between control and treatment groups. (XLSX $41 \mathrm{~kb}$ )

Additional file 6: Figure S2. MA plot. (TIFF $1966 \mathrm{~kb}$ )

\section{Abbreviations}

AA, arbor acres; ADFI, average daily feed intake; ADG, average daily gain; PPAR, peroxisome proliferator-activated receptor; DEGs, differentially expressed genes; FCR, feed conversion ratio; FPKM, fragments per kilobase of transcript per million mapped fragments; GAPDH, glyceraldehyde-3phosphate dehydrogenase; GO, gene ontology; KEGG, Kyoto Encyclopedia of Genes and Genomes; $\mathrm{NH}_{3}$, ammonia; NRC, National Research Council

\section{Acknowledgements}

This work was supported by the China Postdoctoral Science Foundation (No. 2015 M581220), National Key Research and Development Program of China (No. 2016YFD0500501), National Science and Technology Support Program (No. 2012BAD39B01), China Agriculture Research System (No. CARS-42) and 
the Agricultural Science and Technology Innovation Program (No. ASTIP-IAS07) in China.

\section{Funding}

This project was supported by the China Postdoctoral Science Foundation (No. 2015 M581220), National Key Research and Development Program of China (No. 2016YFD0500501), National Science and Technology Support Program (No. 2012BAD39B01), China Agriculture Research System (No. CARS-42) and the Agricultural Science and Technology Innovation Program (No. ASTIPIAS07) in China.

\section{Availability of data and materials}

The data discussed in this publication have been deposited in NCBI's Gene Expression Omnibus (GEO) and are accessible through GEO Series accession number GSE84099 (http://www.ncbi.nlm.nih.gov/geo/query/acc.cgi?acc= GSE84099).

\section{Authors' contributions}

BY participated in the design of the study, performed the statistical analysis and drafted the manuscript. LC participated in the data analysis and helped to draft the manuscript. RQZ and HX carried out the animal experiment. HFZ and RNS conceived of the study, and participated in its design and coordination. All authors read and approved the final manuscript.

\section{Competing interests}

The authors declare that they have no competing interests.

\section{Consent for publication}

Not applicable.

\section{Ethics approval and consent to participate}

Not applicable.

Received: 12 May 2016 Accepted: 21 July 2016

Published online: 11 August 2016

\section{References}

1. Kristensen $\mathrm{HH}$, Wathes $\mathrm{CM}$. Ammonia and poultry welfare: a review. World's Poultry Sci J. 2000;56(03):235-45.

2. Behera SN, Sharma M, Aneja VP, Balasubramanian R. Ammonia in the atmosphere: a review on emission sources, atmospheric chemistry and deposition on terrestrial bodies. Environ Sci Pollution Res Int. 2013;20(11): 8092-131.

3. David B, Mejdell C, Michel V, Lund V, Moe RO. Air Quality in Alternative Housing Systems May Have an Impact on Laying Hen Welfare. Part I-Ammonia. Animals. 2015:5(3):886-96.

4. Dai X-R, Saha CK, Ni J-Q, Heber AJ, Blanes-Vidal V, Dunn JL. Characteristics of pollutant gas releases from swine, dairy, beef, and layer manure, and municipal wastewater. Water Res. 2015;76:110-9.

5. David B, Moe RO, Michel V, Lund V, Mejdell C. Air quality in alternative housing systems may have an impact on laying hen welfare. Part I-Dust. Animals. 2015;5(3):495-511

6. Green AR, Wathes CM, Demmers TG, Clark JM, Xin H. Development and application of a novel environmental preference chamber for assessing responses of laboratory mice to atmospheric ammonia. J Am Assoc Lab Animal Sci JAALAS. 2008;47(2):49-56.

7. Miles DM, Branton SL, Lott BD. Atmospheric Ammonia is Detrimental to the Performance of Modern Commercial Broilers. Poult Sci. 2004;83(10):1650-4.

8. Monfort P, Kosenko E, Erceg S, Canales J-J, Felipo V. Molecular mechanism of acute ammonia toxicity: role of NMDA receptors. Neurochem Int. 2002; 41(2-3):95-102.

9. Braissant O, Mclin VA, Cudalbu C. Ammonia toxicity to the brain. J Inherit Metab Dis. 2013;36(4):595-612.

10. Auron A, Brophy PD. Hyperammonemia in review: pathophysiology, diagnosis, and treatment. Pediatric Nephrol (Berlin, Germany). 2012;27(2): 207-22.

11. Ferrecchia CE, Jensen $K$, Van Andel R. Intracage Ammonia Levels in Static and Individually Ventilated Cages Housing C57BL/6 Mice on 4 Bedding Substrates. J Am Assoc Lab Animal Sci Jaalas. 2014;53(2):146-51.
12. Wei FX, Hu XF, Sa RN, Liu FZ, Li SY, Sun QY. Antioxidant capacity and meat quality of broilers exposed to different ambient humidity and ammonia concentrations. Genet Mol Res. 2014;13(2):3117-27.

13. Piórkowska K, Żukowski K, Nowak J, Połtowicz K, Ropka-Molik K, Gurgul A. Genome-wide RNA-Seq analysis of breast muscles of two broiler chicken groups differing in shear force. Anim Genet. 2015;47(1):68-80.

14. Mutryn MF, Brannick EM, Fu W, Lee WR, Abasht B. Characterization of a novel chicken muscle disorder through differential gene expression and pathway analysis using RNA-sequencing. BMC Genomics. 2015;16(1):399-417.

15. Li T, Wu R, Zhang Y, Zhu D. A systematic analysis of the skeletal muscle miRNA transcriptome of chicken varieties with divergent skeletal muscle growth identifies novel miRNAs and differentially expressed miRNAs. BMC Genomics. 2011;12(1):1-20.

16. Cogburn LA, Wang X, Carre W, Rejto L, Aggrey SE, Duclos MJ, Simon J, Porter TE. Functional genomics in chickens: development of integratedsystems microarrays for transcriptional profiling and discovery of regulatory pathways. Comp Funct Genomics. 2004;5(3):253-61.

17. Picard B, Lebret B, Cassar-Malek I, Liaubet L, Berri C, Le Bihan-Duval E, Hocquette JF, Renand G. Recent advances in omic technologies for meat quality management. Meat Sci. 2015;109:18-26.

18. D'Alessandro A, Zolla L. Meat science: From proteomics to integrated omics towards system biology. J Proteome. 2013;78:558-77.

19. Wang Z, Gerstein M, Snyder M. RNA-Seq: a revolutionary tool for transcriptomics. Nat Rev Genet. 2009;10(1):57-63.

20. Yan Y, Yang N, Cheng HH, Song J, Qu L. Genome-wide identification of copy number variations between two chicken lines that differ in genetic resistance to Marek's disease. BMC Genomics. 2015;16:843.

21. Buermans HPJ, den Dunnen JT. Next generation sequencing technology: Advances and applications. Biochim Biophys Acta (BBA) - Mol Basis Dis. 2014;1842(10):1932-41.

22. Perumbakkam S, Muir WM, Black-Pyrkosz A, Okimoto R, Cheng HH. Comparison and contrast of genes and biological pathways responding to Marek's disease virus infection using allele-specific expression and differential expression in broiler and layer chickens. BMC Genomics. 2013; 14(1):64.

23. Wei FX, Hu XF, Xu B, Zhang MH, Li SY, Sun QY, Lin P. Ammonia concentration and relative humidity in poultry houses affect the immune response of broilers. Genet Mol Res. 2015;14(2):3160-9.

24. Zhang J, Li C, Tang X, Lu Q, Sa R, Zhang H. High Concentrations of Atmospheric Ammonia Induce Alterations in the Hepatic Proteome of Broilers (Gallus gallus): An iTRAQ-Based Quantitative Proteomic Analysis. PLoS One. 2015:10(4):e0123596.

25. Połtowicz K, Doktor J. Effect of slaughter age on performance and meat quality of slow-growing broiler chickens. Ann Anim Sci. 2012;12(4):621-31.

26. da Huang W, Sherman BT, Lempicki RA. Systematic and integrative analysis of large gene lists using DAVID bioinformatics resources. Nat Protoc. 2009; 4(1):44-57.

27. Haas BJ, Papanicolaou A, Yassour M, Grabherr M, Blood PD, Bowden J, Couger MB, Eccles D, Li B, Lieber M. De novo transcript sequence reconstruction from RNA-seq using the Trinity platform for reference generation and analysis. Nat Protoc. 2013;8(8):1494-512.

28. Hillier LW, Miller W, Birney E, Warren W, Hardison RC, Ponting CP, Bork P, Burt DW, Groenen MAM, Delany ME, et al. Sequence and comparative analysis of the chicken genome provide unique perspectives on vertebrate evolution. Nature. 2004:432(7018):695-716.

29. Rekaya R, Sapp RL, Wing T, Aggrey SE. Genetic evaluation for growth, body composition, feed efficiency, and leg soundness. Poult Sci. 2013;92(4):923-9.

30. Ni J-Q. Research and demonstration to improve air quality for the US animal feeding operations in the 21 st century-A critical review. Environ Pollut. 2015;200:105-19.

31. Park JH, Kim IH. Interactive effects of fenugreek (Trigonella foenum-graecum L.) seed extract supplementation and dietary metabolisable energy levels on the growth performance, total tract digestibility, blood profiles, and excreta gas emission in broiler chickens. Animal Product Sci. 2015. http://dx.doi.org/10. 1071/AN14834.

32. Shu G, Liao WY, Feng JY, Yu KF, Zhai YF, Wang SB, Khondowe $P$, Wang $X Q$, Jiang QY. Active immunization of fatty acid translocase specifically decreased visceral fat deposition in male broilers. Poult Sci. 2011;90(11): 2557-64.

33. Davis RV, Lamont SJ, Rothschild MF, Persia ME, Ashwell CM, Schmidt CJ. Transcriptome analysis of post-hatch breast muscle in legacy and modern 
broiler chickens reveals enrichment of several regulators of myogenic growth. PLoS One. 2015;10(3):e0122525.

34. Guo J, Shu G, Zhou L, Zhu X, Liao W, Wang S, Yang J, Zhou G, Xi Q, Gao P, et al. Selective transport of long-chain fatty acids by FAT/CD36 in skeletal muscle of broilers. Animal. 2013;7(03):422-9.

35. Tarhda Z, Semlali O, Kettani A, Moussa A, Abumrad NA, Ibrahimi A. Three Dimensional Structure Prediction of Fatty Acid Binding Site on Human Transmembrane Receptor CD36. Bioinformatics Biol Insights. 2013;7:369-73.

36. McFarlan JT, Yoshida Y, Jain SS, Han X-X, Snook LA, Lally J, Smith BK, Glatz JFC, Luiken JJFP, Sayer RA, et al. In Vivo, Fatty Acid Translocase (CD36) Critically Regulates Skeletal Muscle Fuel Selection, Exercise Performance, and Training-induced Adaptation of Fatty Acid Oxidation. J Biol Chem. 2012; 287(28):23502-16.

37. Joseph R, Poschmann J, Sukarieh R, Too PG, Julien SG, Xu F, Teh AL, Holbrook JD, Ng KL, Chong YS, et al. ACSL1 Is Associated With Fetal Programming of Insulin Sensitivity and Cellular Lipid Content. Mol Endocrinol. 2015;29(6):909-20.

38. Cui H, Liu R, Zhao G, Zheng M, Chen J, Wen J. Identification of differentially expressed genes and pathways for intramuscular fat deposition in pectoralis major tissues of fast-and slow-growing chickens. BMC Genomics. 2012;13(1): $1-12$.

39. Wu Q, Ortegon AM, Tsang B, Doege H, Feingold KR, Stahl A. FATP1 Is an Insulin-Sensitive Fatty Acid Transporter Involved in Diet-Induced Obesity. Mol Cell Biol. 2006;26(9):3455-67.

40. Melo C, Gallardo D, Quintanilla R, Zidi A, Castelló A, Díaz I, Amills M, Pena RN. An association analysis between polymorphisms of the pig solute carrier family 27A (SLC27A), member 1 and 4 genes and serum and muscle lipid traits. Livest Sci. 2013;152(2-3):143-6.

41. Cui HX, Zheng MQ, Liu RR, Zhao GP, Chen JL, Wen J. Liver dominant expression of fatty acid synthase (FAS) gene in two chicken breeds during intramuscular-fat development. Mol Biol Rep. 2011;39(4):3479-84.

42. Zhao C, Tian F, Yu Y, Liu G, Zan L, Updike MS, Song J. miRNA-dysregulation associated with tenderness variation induced by acute stress in Angus cattle. J Animal Sci Biotechnol. 2012;3(1):1.

43. Sackett BAM, Fronting GW, Deshazer JA, Struwe FJ. Effect of Gaseous Preslaughter Environment on Chicken Broiler Meat Quality. Poult Sci. 1986; 65(3):511-9.

44. Maltin C, Balcerzak D, Tilley R, Delday M. Determinants of meat quality: tenderness. Proc Nutr Soc. 2003;62(02):337-47.

45. Quarles CL, Kling HF. Evaluation of Ammonia and Infectious Bronchitis Vaccination Stress on Broiler Performance and Carcass Quality. Poult Sci. 1974;53(4):1592-6.

46. Pearce KL, Rosenvold K, Andersen HJ, Hopkins DL. Water distribution and mobility in meat during the conversion of muscle to meat and ageing and the impacts on fresh meat quality attributes - A review. Meat Sci. 2011; 89(2):111-24.

47. Razinia Z, Baldassarre M, Cantelli G, Calderwood DA. ASB2a, an E3 Ubiquitin Ligase Specificity Subunit, Regulates Cell Spreading and Triggers Proteasomal Degradation of Filamins by Targeting the Filamin Calponin Homology 1 Domain. J Biol Chem. 2013;288(44):32093-105.

48. Davey JR, Watt Kl, Parker BL, Chaudhuri R, Ryall JG, Cunningham L, Qian H, Sartorelli V, Sandri M, Chamberlain J, et al. Integrated expression analysis of muscle hypertrophy identifies ASB2 as a negative regulator of muscle mass. JCl Insight. 2016;1(5):e85477.

49. Bower $\mathrm{Nl}$, Johnston IA. Discovery and characterization of nutritionally regulated genes associated with muscle growth in Atlantic salmon. Physiol Genomics. 2010;42A(2):114-30.

50. Bosma M, Hesselink MKC, Sparks LM, Timmers S, Ferraz MJ, Mattijssen F, van Beurden D, Schaart G, de Baets MH, Verheyen FK, et al. Perilipin 2 Improves Insulin Sensitivity in Skeletal Muscle Despite Elevated Intramuscular Lipid Levels. Diabetes. 2012;61(11):2679-90.

51. Conte M, Vasuri F, Trisolino G, Bellavista E, Santoro A, Degiovanni A, Martucci E, D'Errico-Grigioni A, Caporossi D, Capri M, et al. Increased Plin2 expression in human skeletal muscle is associated with sarcopenia and muscle weakness. PLoS One. 2013;8(8):e73709.

52. Davoli R, Gandolfi G, Braglia S, Comella M, Zambonelli P, Buttazzoni L, Russo V. New SNP of the porcine Perilipin 2 (PLIN2) gene, association with carcass traits and expression analysis in skeletal muscle. Mol Biol Rep. 2010;38(3): 1575-83.

53. Gandolfi G, Mazzoni M, Zambonelli P, Lalatta-Costerbosa G, Tronca A, Russo V, Davoli R. Perilipin 1 and perilipin 2 protein localization and gene expression study in skeletal muscles of European cross-breed pigs with different intramuscular fat contents. Meat Sci. 2011;88(4):631-7.

54. Xing K, Zhu F, Zhai L, Liu H, Wang Z, Hou Z, Wang C. The liver transcriptome of two full-sibling Songliao black pigs with extreme differences in backfat thickness. J Animal Sci Biotechnol. 2014;5(1):1-9.

55. Zhao GP, Cui HX, Liu RR, Zheng MQ, Chen JL, Wen J. Comparison of breast muscle meat quality in 2 broiler breeds. Poult Sci. 2011;90(10):2355-9.

56. Zhao GP, Chen JL, Zheng MQ, Wen J, Zhang Y. Correlated Responses to Selection for Increased Intramuscular Fat in a Chinese Quality Chicken Line. Poult Sci. 2007;86(11):2309-14.

57. Lu Q, Wen J, Zhang H. Effect of Chronic Heat Exposure on Fat Deposition and Meat Quality in Two Genetic Types of Chicken. Poult Sci. 2007;86(6): 1059-64.

58. Zhao C, Tian F, Yu Y, Luo J, Hu Q, Bequette BJ, Vi RLB, Liu G, Zan L, Updike MS, et al. Muscle transcriptomic analyses in Angus cattle with divergent tenderness. Mol Biol Rep. 2012;39(4):4185-93.

\section{Submit your next manuscript to BioMed Central and we will help you at every step:}

- We accept pre-submission inquiries

- Our selector tool helps you to find the most relevant journal

- We provide round the clock customer support

- Convenient online submission

- Thorough peer review

- Inclusion in PubMed and all major indexing services

- Maximum visibility for your research

Submit your manuscript at www.biomedcentral.com/submit
(O) BioMed Central 